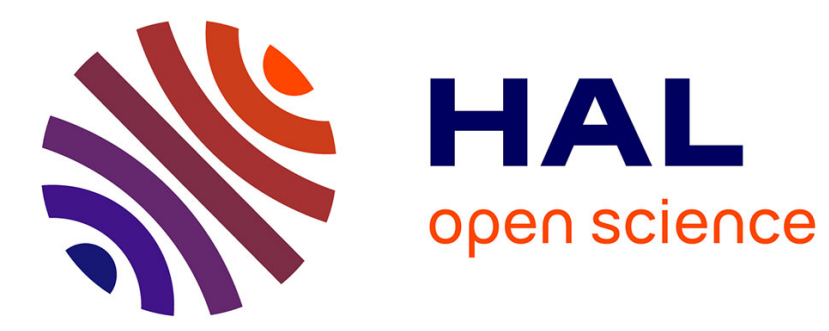

\title{
La Nature, les territoires et le politique en Afrique du Sud
}

Frédéric Giraut, Sylvain Guyot, Myriam Houssay-Holzschuch

\section{To cite this version:}

Frédéric Giraut, Sylvain Guyot, Myriam Houssay-Holzschuch. La Nature, les territoires et le politique en Afrique du Sud. Annales. Histoire, Sciences sociales, 2005, 4, pp.695-717. hal-00185655

\section{HAL Id: hal-00185655 \\ https://hal.science/hal-00185655}

Submitted on 6 Nov 2007

HAL is a multi-disciplinary open access archive for the deposit and dissemination of scientific research documents, whether they are published or not. The documents may come from teaching and research institutions in France or abroad, or from public or private research centers.
L'archive ouverte pluridisciplinaire HAL, est destinée au dépôt et à la diffusion de documents scientifiques de niveau recherche, publiés ou non, émanant des établissements d'enseignement et de recherche français ou étrangers, des laboratoires publics ou privés. 


\title{
La Nature au service du politique en Afrique du Sud ${ }^{1}$
}

\author{
Frédéric GIRAUT, \\ UMR Pacte/Territoires, Université Joseph Fourier ${ }^{2}$ \\ Sylvain GUYOT \\ UMR Pacte/Territoires, Université Pierre Mendès-France ${ }^{3}$ \\ Myriam HOUSSAY-HOLZSCHUCH, \\ UMR Géographie-cités/Géophile, ENS Lettres et Sciences humaines ${ }^{4}$
}

\begin{abstract}
Résumé : Le traitement de la nature dans les colonies de peuplement prend en Afrique du Sud une dimension particulière. La gestion de la nature y fut remarquablement instrumentalisée dans le cadre d'une ingénierie territoriale de la ségrégation coloniale puis de l'apartheid, au point d'être l'une des pièces maîtresses des dispositifs territoriaux et de l'idéologie qui les sous-tendait. Dans la nouvelle Afrique du Sud, elle constitue l'un des terrains privilégiés du raccommodage socio-spatial, et ceci à trois niveaux. Au niveau local avec le développement de formes participatives communautaires de gestion et d'appropriation qui ne vont pas sans poser de problème avec une municipalisation qui privilégie la démocratie représentative ; au niveau national avec un certain consensus autour de la promotion et la restauration d'un patrimoine naturel autochtone d'où l'on doit extirper les plantes allochtones; et au niveau international avec la transformation des parcs frontaliers en des objets internationaux de développement, les transfrontier peace parks.
\end{abstract}

Mots-clefs : Afrique du Sud, nature, environnement, territoire, ségrégation, conservation, colonisation, instrumentalisation, apartheid.

Abstract: In South Africa, nature management has been instrumentalised for spatial engineering during the colonial and apartheid eras. In the new South Africa, 'Nature' has remained an essential tool for repairing the socio-spatial fabric. At a local level, nature management has allowed new, participative, forms of administration to develop, even if they are sometimes inconsistent with new, democratic, municipal structures. At a national level, a consensus for the preservation of an indigenous natural heritage has been agreed upon and "alien plants" have become an enemy to destroy. At an international level, transfrontier peace parks planned for development have replaced former natural parks used as military buffer zones.

Keywords: South Africa, nature, environment, territory, segregation, conservation, colonisation, instrumentalisation, apartheid.

\section{Introduction}

\footnotetext{
${ }^{1}$ Nous remercions Benoît Rey-Soulingeas et Stéphane Vermeulin, qui nous ont aidé à amorcer cette réflexion.

${ }^{2}$ IGA Cités des territoires, 14 bis avenue Marie Reynoard, 38100 Grenoble. frederic.giraut@ujf-grenoble.fr

${ }^{3}$ Université Pierre Mendès-France, ARSH, BP47, 38040 Grenoble cedex. sylvainafrica@free.fr

4 École Normale Supérieure Lettres et Sciences humaines, 15 parvis Descartes, BP 7000, 69342 Lyon cedex 07. Myriam.Houssay@normalesup.org
} 
Le parc naturel de Hluhluwe-Umfolozi, au nord de la province sud-africaine du KwaZulu-Natal, est l'une des zones protégées les plus anciennes, les plus connues et les plus emblématiques du pays. Il est dominé par le haut-lieu de Hilltop, qui regroupe les hébergements les plus prestigieux, un restaurant panoramique et, depuis le début des années 2000, un panneau d'information présentant les origines de l'aire protégée. La réserve, délimitée depuis 1895 par les colonisateurs britanniques, y est replacée dans un espace-temps africain : elle s'étend, dit ce panneau, sur les anciennes réserves de chasse du grand roi zoulou Shaka (1785-1828) et aurait servi de lieu d'initiation aux jeunes gens des environs depuis des temps « immémoriaux ».

Ce type de discours révèle d'une part la nécessité, dans l'Afrique du Sud postapartheid, de légitimer et d' " africaniser » la conservation de la nature en l'inscrivant dans la longue durée de l'histoire précoloniale et dans des logiques régionales et locales d'implication des populations. D'autre part, il montre «en creux » à quel point la vision dominante de la nature, telle qu'elle est présentée et diffusée, s'inscrit dans une idéologie d'inspiration européenne : la réalité des représentations et des pratiques locales reste, encore aujourd'hui, peu connue scientifiquement. De plus, étant le fait de communautés dominées, elles nous sont largement transmises via les discours dominants ${ }^{5}$.

Les espaces naturels protégés sud-africains associent richesse écologique, ressource économique et prestige. En même temps, ces espaces sont hérités de l'époque coloniale et d'un modèle spatial caractérisé par une domination blanche sans partage. Ils sont donc un des enjeux politiques contemporains de l'Afrique du Sud post-apartheid. Réciproquement, ils ont été d'emblée utilisés par l'État colonial pour agir sur l'espace et sur la société. Cette instrumentalisation s'est poursuivie sous des formes différentes et complémentaires malgré les changements politiques majeurs que le pays a connu au XXe siècle : le régime d'apartheid a renforcé ce dispositif territorial en faveur de son projet de ségrégation systématique ; de manière plus surprenante, le gouvernement démocratique actuel utilise les espaces naturels pour construire le développement d'une nation. La Nature sud-africaine est donc mise au service du politique.

\section{Instrumentalisations politiques de la nature}

Dans le contexte scientifique d'un retour critique sur la notion de nature, voire d'une déconstruction des discours environnementalistes ${ }^{6}$, le cas sud-africain permet d'appréhender la construction sociale de l'idée de nature dans un contexte environnemental subtropical et un double contexte culturel, celui de l'Afrique noire et celui d'une colonie de peuplement (settler society). De plus, la question de la nature y a été instrumentalisée et politisée au point d'être consubstantielle à un projet politique et spatial trop fameux, celui de la ségrégation et de l'apartheid.

\section{Modernité / post-modernité : temporalités sud-africaines}

\footnotetext{
${ }^{5}$ Ainsi, le missionnaire écossais Robert Moffat estime que le contrôle de l'environnement est une caractéristique différenciant les Chrétiens européens, des Africains. Son ouvrage de 1842, Missionnary Labours and Scene in Southern Africa, souligne le contraste entre le paysage ordonné et aménagé autour de la mission et la wildness africaine.

${ }^{6}$ Philippe Pelletier, L’imposture écologiste, Montpellier, Reclus, Géographiques, 1993.
} 
La conception que l'on se fait en Afrique du Sud de la nature a, bien évidemment, évolué selon les époques et les contextes socio-politiques. En conséquence, les types d'instrumentalisation comme leurs transcriptions spatiales sont divers, même si l'instrumentalisation de la nature à des fins politiques est récurrente.

En établir les temporalités nécessite de prendre en compte une histoire qui échappe partiellement aux distinctions classiques dans cette partie du monde, entre époque précoloniale, coloniale et post-coloniale. En effet, si l'identification de la période précoloniale pose peu problème, la césure entre colonial et post-colonial est particulièrement difficile à définir ${ }^{7}$. La colonisation a débuté avec l'installation en 1652 de Jan van Riebeeck dans la péninsule du Cap et la diffusion progressive du peuplement et de la domination européens dans le sous-continent. Quand s'est-elle terminée ? En 1852, lors de la création de l'État libre d'Orange, république afrikaner ${ }^{8}$ indépendante ? En 1910, avec la naissance de l'Union sudafricaine et son indépendance politique vis-à-vis de la métropole britannique ? En 1948, avec l'accession au pouvoir des Afrikaners aux dépens des anglophones ? En 1976, lors de la pseudo-indépendance du Transkei, réserve noire, présentée comme de la décolonisation par le gouvernement d'apartheid ? En 1994, lors de la première élection au suffrage universel, celle de Nelson Mandela à la présidence ? Le régime d'apartheid et ses manipulations politiques brouillent donc la clarté du passage entre époque coloniale et époque post-coloniale.

En revanche, si le traitement de la nature est le principal critère envisagé, la coupure entre modernité et post-modernité apparaît bien comme pertinente, en Afrique du Sud comme ailleurs. Nous adopterons ici une définition de la modernité comme un ensemble de représentations, de techniques et de pratiques privilégiant une approche rationalisante et de grandes oppositions (Nature / Culture, civilisé / sauvage...). Dans ce cadre, le traitement de la nature en Afrique du Sud entre 1652 et 1994 est effectivement caractérisé par une logique binaire et entre ainsi dans des pratiques de mise en ordre et de rationalisation du territoire. Dans les diverses définitions de la post-modernité ${ }^{9}$, nous choisissons de privilégier quelques

\footnotetext{
${ }^{7}$ Myriam Houssay-Holzschuch, Mythologies territoriales en Afrique du Sud : un essai de géographie culturelle, Paris, CNRS Éditions, Espaces et Milieux, 1995.

${ }^{8}$ La dénomination de groupes « raciaux» en Afrique du Sud, et plus particulièrement dans l'Afrique du Sud post-apartheid, est un problème ardu doublé d'un sujet politiquement sensible. La classification raciale en vigueur sous l'apartheid comprenait :
}

- $\quad$ Les «Blancs » (Sud-africains ayant des origines européennes), parmi lesquels on distinguait :

- Les Afrikaners, descendants des colons hollandais installés dans le pays depuis le XVIIe siècle et parlant l'Afrikaans. Le terme Afrikaner signifie «Africain» en hollandais. Il a par conséquent une connotation politique, soulignant l'identité africaine de ce groupe de population.

- Les Sud-africains anglophones.

○ D'autres immigrants - souvent arrivés plus tardivement - d'origine européenne, tels que les Grecs, les Portugais...

- Les «Indiens », Sud-africains d'origine indienne dont les ancêtres sont souvent venus comme travailleurs sous contrat ou comme passagers libres, notamment à la fin du XIXe siècle et au début du XXe siècle ;

- $\quad$ Les «Africains » (dénommés auparavant « Natifs » ou « Bantous »), Sud-africains d'origine africaine ;

- Les « Métis » ou «Colorés » (Coloureds), groupe hétérogène incluant les personnes ayant des origines mélangées et celles qui ne rentrent pas dans les catégories mentionnées ci-dessus.

Cette classification a joué un rôle important dans la construction identitaire. Tout en condamnant le système qui l'a conçue, les chercheurs l'emploient toujours abondamment. Dans cet essai, même si nous sommes conscients des implications politiques de chaque terme, nous suivrons les conventions usuelles et emploieront les catégories ci-dessus telles qu'elles sont comprises dans le contexte sud-africain.

9 Géographie et Cultures, numéro spécial «La postmodernité. Visions anglophone et francophone », $\mathrm{n}^{\circ} 31$, 1999 ; L'espace géographique, Débat : «Le postmodernisme en géographie », nº1, 2004 ; David Harvey, The 
aspects : la reconnaissance, voire la recherche de la diversité, le jeu entre des logiques multiples, la transformation des dispositifs rationnels au profit du spécifique (local) et de l'intégré (global). Dans ce cadre, la notion d'environnement vient compléter et modifier celle de nature ${ }^{10}$. Depuis 1994, l'Afrique du Sud post-apartheid se situe dans cette perspective, de manière explicite voire revendiquée ${ }^{11}$.

\section{Modalités d'instrumentalisation}

L'instrumentalisation politique de la nature est un des outils privilégié de l'État sudafricain depuis sa constitution. Elle se fait selon différents types, chaque période - moderne ou post-moderne -inventant et privilégiant certaines modalités. Néanmoins, ces différentes modalités sont liées les unes aux autres et doivent être comprises dans le cadre d'un système plus général, celui de l'action de l'État. Ce système d'action semble remarquable par sa continuité entre période et post-moderne - même si la légitimité démocratique de l'État sudafricain actuel a changé la nature de cet État. En effet, les différentes modalités d'instrumentalisation politique de la nature s'accumulent par «sédimentation » sans que les nouvelles remplacent strictement les plus anciennes. En ce sens, on retrouve la notion de postmodernité, qui s'inscrit en continuité de la modernité.

La première modalité d'instrumentalisation identifiable historiquement concerne la manière dont la nature est mobilisée pour fabriquer des représentations sociales. Celles-ci concernent plusieurs domaines : des types de populations humaines et d'espèces animales, des espaces. On peut individualiser quatre objets principaux de ces représentations : 1' «indigène » (Native), le colon européen vu dans son parangon britannique, l'animal, l'Afrique. Ces représentations forment aussi un système, caractérisé par les oppositions binaires typiques de la modernité. Indigène et colon s'opposent évidemment mais cette structure manichéenne est valable aussi pour la catégorie des animaux, dans laquelle on distingue les nuisibles des grands mammifères. Le prestige accordé à ces derniers leur permet, suivant les périodes, de figurer comme trophées de chasse sur les murs de l'aristocratie ou d'être protégés.

Ces représentations sont instrumentalisées à leur tour à des fins politiciennes : elles sont utilisées dans les discours politiques à visée électoraliste, basés sur le « us-versus-them ». Avant 1994, les représentations de l'indigène et du colon tiennent une large place dans les discours de justification de la ségrégation et de l'apartheid. Ils associent étroitement la nature à la prétendue sauvagerie africaine comme en témoigne le proverbe "You can take the African out of the bush, but you can't take the bush out of the African $»^{12}$.Aujourd'hui, les représentations de l'animal et de l'Afrique soutiennent une rhétorique à la fois conservationniste et conservatrice (cf. infra).

Cependant, la modalité principale est de faire de la nature un outil essentiel de la gestion sociale. Cela est pratiqué de deux manières, à des fins de division ou au profit d'un projet national unificateur. En effet, la création des parcs naturels permet dans le même temps d'expulser et d'exclure des Africains de leurs terres « ancestrales » et de réserver des espaces de récréation hédonique à des populations blanches en recherche d'exotisme. De plus, ils sont utilisés comme des outils dans la construction symbolique de l'unité nationale : dans les

Condition of Postmodernity, Oxford, Blackwell, 1988 ; Ed Soja, Postmodern Geographies, The Reassertion of Space in Critical Social Theory, London, Verso, 1989.

${ }^{10}$ Augustin Berque, Médiance : de milieux en paysages, Montpellier, Reclus, Géographiques, 1990.

${ }^{11}$ Le Bill of Rights, les discours sur la Renaissance africaine en sont deux exemples.

${ }^{12}$ Mark Behr, The Smell of Apples. London, Abacus, 1995. 
phases de réconciliation suivant des conflits ayant profondément déchiré le tissu social - que ce soit après la guerre des Boers pour unir Anglais et Afrikaners ou depuis 1994 rassembler Noirs et Blancs -, l'État agit sur les espaces naturels, et en particulier sur les parcs nationaux, pour en faire des symboles d'un projet national. Il s'agit dans la période moderne du projet de domination blanche, dans la période post-moderne d'un projet de développement durable (sur les plans économiques, sociaux et écologiques).

L'action de l'État passe également par une instrumentalisation territoriale de la nature. Le contrôle de l'espace est assuré par un strict zonage, délimitant des aires fonctionnelles sous couvert de discours sur la nature : un premier discours, préservationniste, va servir à séparer les zones d'exploitation de zones protégées. Un deuxième discours, élitiste, réservera l'usage des zones naturelles aux Blancs. Un dernier discours, politique, utilise les espaces protégés pour introduire les réformes administratives et gestionnaires souhaitées.

L'instrumentalisation territoriale s'envisage aussi à une autre échelle, supra-nationale, et qui consacre l'utilisation géopolitique des espaces naturels en Afrique du Sud. Celle-ci persiste dans des contextes opposés, d'une géopolitique de guerre ou de paix, de puissance ou de coopération multilatérale. Ainsi, durant l'apartheid, les parcs naturels frontaliers ont servi à des fins militaires.

Enfin, les programmes post-apartheid de réduction des inégalités sociales se servent des espaces naturels comme lieux de développement : ils doivent générer des revenus et donc avoir une rentabilité économique permettant d'entraîner les régions environnantes. Par le biais du tourisme international, ils sont vus comme un moyen d'intégrer l'Afrique du Sud au reste du monde et de profiter de la mondialisation. Enfin, ils servent de lieu d'expérimentation de nouvelles formes politiques, participatives, que l'on souhaite plus efficaces pour le développement local.

Ainsi, ces différentes modalités d'instrumentalisation politique de la nature peuvent être identifiées dans l'action de l'État. Si certaines sont plus actives que d'autres à une période donnée, elles s'ajoutent les unes aux autres sans jamais s'annuler complètement. Les champs sociaux, territoriaux et géopolitique restent trois modalités centrales à l'époque moderne comme à l'époque post-moderne. Cette sédimentation et cette continuité font du domaine de la nature un enjeu d'une mémoire contestée : parler de la nature, c'est interpréter le passé dans lequel cette instrumentalisation s'est construite.

\section{Une généalogie de l'instrumentalisation de la nature}

L'instrumentalisation politique de la nature est donc ancienne et continue. En même temps, sa persistance sous des régimes extrêmement différents pose en elle-même des problèmes politiques de premier ordre qu'il faut résoudre dans le cadre de la construction nationale contemporaine. Un détour historique permet d'en comprendre mieux les enjeux actuels en en mettant au jour la généalogie coloniale, ségrégative et d'apartheid.

\section{L'ordre colonial et ses « réserves » : 1890-1920s}

La fin du XIXe siècle va voir l'éclosion de l'idée de parcs et de réserves de faune et de flore dans l'ensemble des colonies de peuplement d'origine britannique : Yellowstone aux États-Unis en 1872, le Royal National Park dans les environs de Sidney en Australie en 1879 
et les réserves de faune de Hluhluwe et Umfolozi au Zululand en 1895. Le principe du zonage fonctionnel et la figure spatiale de la séparation comme moyen de régulation géopolitique, marquant particulièrement les pratiques britanniques, s'appliquent donc aussi au domaine du «naturel ». Mieux, le naturel strictement délimité, va renforcer le zonage fonctionnel et être utilisé comme moyen de séparation. C'est le début de l'instrumentalisation de la nature dans un projet politique et territorial global.

Cette ingénierie territoriale commune aux colonies de peuplement anglo-saxonnes a cependant des spécificités lorsqu'elle s'applique aux colonies africaines. En effet, si l'on cherche avant tout à préserver un paysage grandiose et fragile aux Etats-Unis, le point de départ de la gestion sud-africaine de la nature est l'animal. Gênant et convoité en même temps, on veut l'enfermer dans des réserves. Il est ainsi exclu de l'espace productif européen. En même temps, il est institué comme objet de loisir, via la chasse sportive. Celle-ci est un privilège britannique et une marque de supériorité : la recherche et l'exposition du trophée incarnent le triomphe de l'Anglo-Saxon face à la sauvagerie africaine. Elle s'oppose aux pratiques des autres populations sud-africaines, celle des Africains pour qui le gibier est un élément de subsistance et celle des Afrikaners, pour qui le produit de la chasse est une ressource commercialisable ${ }^{13}$.

La délimitation de réserves de chasse en Afrique du Sud ne recouvre pas que des fonctions cynégétiques et esthétiques. Elle participe d'une mutation économique majeure des campagnes sud-africaines. Les populations locales, privées de l'accès à des ressources de gibier essentielles dans l'économie traditionnelle, sont poussées à aller travailler dans les mines. La géographie de ces réserves est un élément supplémentaire pour expliquer ces conséquences sur les populations : il s'agit d'un ensemble d'isolats au sein de l'espace rural qui jouxtent de fortes concentrations de populations africaines (cf. figure 1).

\footnotetext{
${ }^{13}$ Jane Carruthers, "Nationhood and national parks: comparative examples from the post-imperial experience" in T. Griffiths et L. Robin (dir), Ecology \& Empire. Environmental History of Settler Societies, Pietermaritzburg, University of Natal Press, 1997 pp. 125-138.
} 


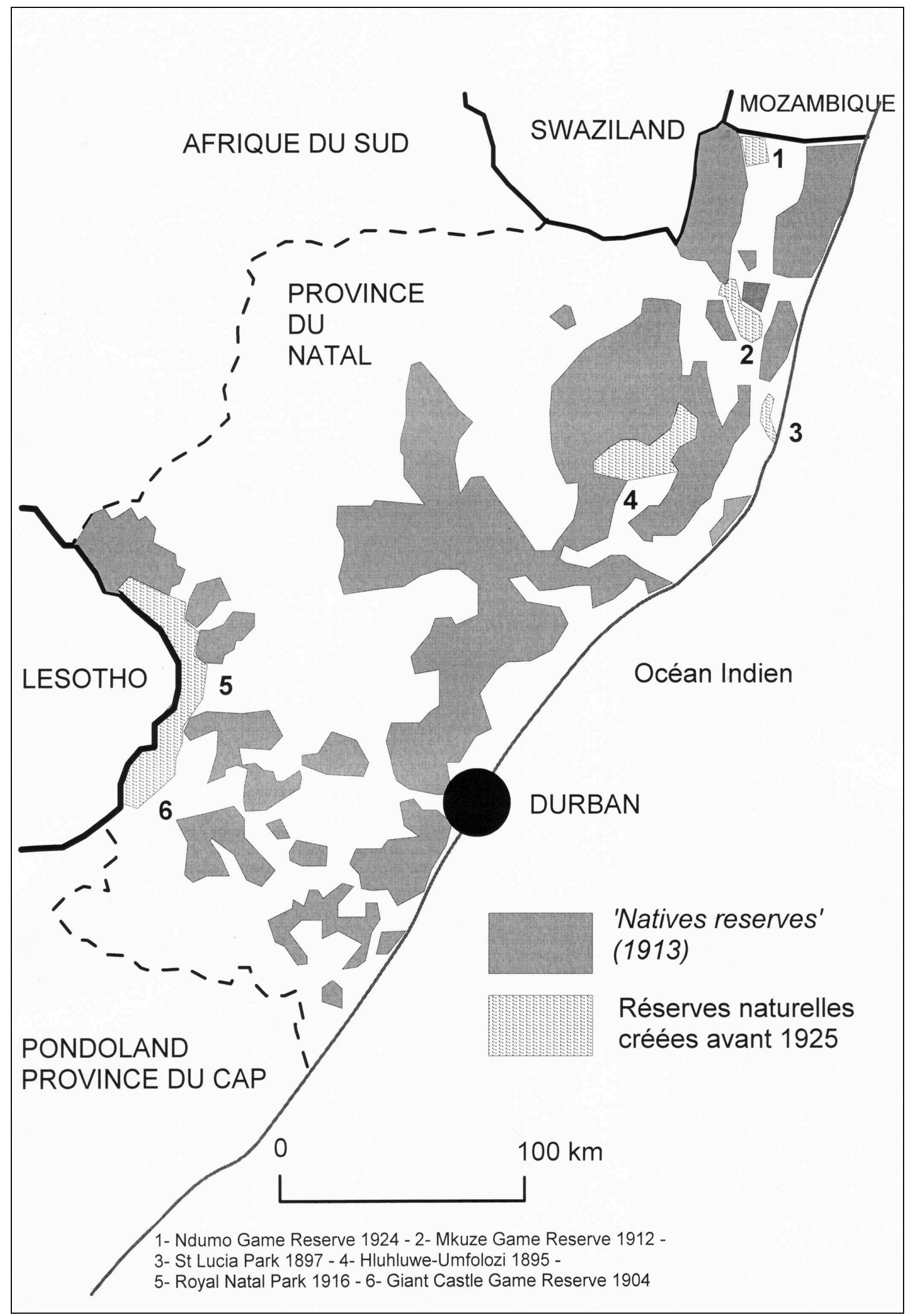


Figure 1 : L'ingénierie territoriale. Réserves naturelles et réserves africaines au Natal en $1936^{14}$.

Ces réserves sont constitutives d'un dispositif territorial plus vaste, qui comprend également les réserves indigènes (Native Reserves - le terme est le même pour les espaces assignés aux «indigènes » et dévolus à la nature) et le domaine approprié par les colons (Crown Land). Ce dispositif est mis en place au fur et à mesure de la conquête de l'espace bantou et il est l'un des moyens du contrôle colonial. Il commencera à être théorisé au milieu du XIXe siècle par Theophilus Shepstone dans la Colonie du Natal avec le location system et sera en quelque sorte standardisé et étendu à l'ensemble de l'Afrique du Sud en 1913 avec le Native Land Act. Le partage très inégal des terres connaît alors une traduction territoriale duale $^{15}$. Aux colons, un vaste domaine organisé en provinces et disposant d'institutions municipales qui intègre la grande majorité de l'espace sud-africain et notamment les villes et la quasi-totalité des terres les plus fertiles ou disposant de ressources minières ; aux indigènes, un archipel de réserves ${ }^{16}$ densément peuplées où règnent la propriété collective et les autorités tribales. La géographie administrative du pays est donc profondément hétérogène et les différences de statut juridique et de mode d'administration correspondent, au-delà de l'état de l'occupation coloniale, à des vocations irrémédiablement opposées. D'un côté, les terres de la Couronne sont vouées à une exploitation économique rationnelle et moderne, par l'agriculture commerciale, l'exploitation minière et le développement industriel et urbain; de l'autre, les réserves indigènes sont le cadre de la reproduction des sociétés africaines " traditionnelles », par l'agriculture de subsistance et la permanence des encadrements. Dès lors, les conditions de la dépendance économique et du contrôle d'une main-d'œuvre captive sont inscrites dans l'organisation territoriale de l'Union sud-africaine.

Dans cette nouvelle géographie, les game reserves sont des enclaves de nature. Elles sont soustraites à l'espace des Africains pour offrir à la colonie de peuplement des espaces de loisir. Ainsi, la réserve de chasse de Hluhluwe, la plus ancienne d'Afrique australe, créée en 1895, est d'abord gérée par l'administration britannique du Zululand, puis directement par l'administration de la Colonie du Natal à partir de 1897.

Au-delà du transfert administratif (passage du domaine de l'administration indigène à l'administration de la colonie de peuplement) on peut voir dans la position, en quelque sorte intermédiaire, de ces réserves de chasse une fonction de zone tampon entre le domaine rural des colons et celui des Bantous. C'est dans ce cadre que certains auteurs soulignent la fonction de protection assignée à ces espaces. Ainsi Shirley Brooks ${ }^{17}$ identifie à partir du cas de Hluhluwe deux logiques dans la politique des game reserves : celle déjà évoquée de la chasse coloniale qu'elle estime relever d'une logique de «pursuit » (perpétuation de la faune et de la chasse); celle de la protection contre les animaux sauvages et les épizooties dont ils pourraient être porteurs (ici la nagana, maladie endémique qui frappe les troupeaux du Zululand) qu'elle qualifie de logique de «containment » (réduction et enfermement).

\footnotetext{
${ }^{14}$ Source : KZN Wildlife et Philippe Gervais-Lambony, Territoires citadins. 4 villes africaines, Paris, Belin, Mappemonde, 2003.

${ }^{15}$ J. Lambert, « The titihoya does not cry here any more': the crisis in the homestead economy in colonial Natal", in S. Dovers, R. Edgecombe et B. Guest (dir.), South Africa's Environmental History Cases and Comparisons, Athens, Ohio University Press, coll. Ecology and History, 2002, pp. 48-60.

${ }^{16}$ Moins de 10 millions d'hectares, soit moins de 8,5\% du territoire. Elles regroupent environ $60 \%$ de la population noire de l'Union.

${ }^{17}$ S. Brooks, Changing Nature: A Critical Historical Geography of the Umfolozi and Hluhluwe Game Reserves, Zululand, 1887 to 1947, PhD Thesis, Dpt of Geography, Queens University (Canada), 2001.
} 


\section{Vers le Green apartheid : 1920s-1994}

Au début du XXe siècle, la chasse de loisirs a peu à peu remplacé la chasse utilitaire. La diminution des ressources en gibier sauvage, et son rôle présumé dans la contamination du bétail domestique a progressivement conduit à passer d'une stratégie de préservation utilitaire à une stratégie de conservation ${ }^{18}$.

La Convention pour la protection de la faune et de la flore en Afrique (Agreement for the Protection of the Fauna and Flora of Africa), réunie en 1933 à Londres, consacre ce passage à la conservation. Cette convention formalise en fait au niveau international l'institutionnalisation et la territorialisation progressive de la protection ainsi que la rupture avec la fonction ou la pratique cynégétique ${ }^{19}$.

Le tourisme s'impose alors comme objectif prioritaire pour les parcs nationaux. Le Royaume-Uni a inventé toutes les formes de tourisme, il a cultivé l'art de voyager. Le parc naturel représente donc un lieu très normé du tourisme exotique mis à la disposition des clientèles britanniques et des résidents des colonies de peuplement d'Afrique orientale et australe. Il incarne aussi la grandeur de l'Empire avec ses multiples lieux sauvages de dépaysement rendus enfin fréquentables par la qualité et la rigueur des aménagements mis en place. En effet, le parc naturel britannique est un modèle de planification qui allie le confort (lodges, domestiques) à l'exploration sauvage (les débuts du safari... $)^{20}$.

\section{Parcs et projet national}

En Afrique du Sud, l'émergence de la notion de parc national s'effectue dans les années 1920 avec la création du Parc National Kruger et la même année, 1926, celle du South African National Parks Board chargé de la gestion de ces espaces, en tant qu'attributs de 1'Etat-Nation. Suivront en 1931 : l'Addo Elephant National Park, le Kalahari Gemsbok National Park et le Bontebok National Park puis en 1937 le Moutain Zebra National Park. On le voit, il s'agit là de protéger une espèce animale emblématique en lui délimitant un territoire (cf. figure 2).

Le Parc National Kruger ${ }^{21}$ relève lui d'une logique différente, qui doit être rapprochée du contexte politique sud-africain... Les anglophones, qui le constituent à partir de deux game

\footnotetext{
${ }^{18}$ J. M. MacKenzie, The Empire of Nature. Hunting, Conservation and British Colonialism, Manchester, Manchester University Press,1988; Estienne Rodary, Les espaces naturels : l'aménagement par la participation? Mise en réseau et territorialisation des politiques de conservation de la faune en Zambie et au Zimbabwe, Thèse de Doctorat, Université d'Orléans, 2001.

${ }^{19}$ Signature à Londres dès 1900 d'une Convention for the Preservation of Wild Animals, Birds and Fish in Africa : création en 1903 de la Society for the Fauna of the Empire, active jusqu'à la seconde guerre mondiale ; instauration d'une Consultative Commission for the Protection of Nature (1913-1918) puis d'un Bureau International pour la Conservation de la Nature créé en 1928. Voir E. Rodary et C. Castellanet C., "Les trois temps de la conservation" in E. Rodary, C. Castellanet et G. Rossi (dir.), Conservation de la nature et développement, l'intégration impossible ?, Paris, GRET - Karthala, 2004, pp. 5-44.

${ }^{20}$ Njabulo Ndebele, "Game lodges and leisure colonialists" in H. Judin et I. Vladislavic (dir.), Blank Architecture, apartheid and after, Cape Town, David Philip Publishers, 1998, pp. 119-123 ; Sylvain Guyot, «Derrière le masque de l'écotourisme, le politique : conservation et discrimination territoriale en Afrique du Sud », Revue Tiers-Monde, t. XLV, n²178, 2004, pp 341-363.

${ }^{21}$ Jane Carruthers, The Kruger National Park: a social and political history, Pietermaritzburg: University of Natal Press, 1995.
} 
reserves, lui donnent le nom d'une grande figure afrikaner ${ }^{22}$ pour affirmer la dimension nationale (blanche) de l'Union alors même qu'un nationalisme afrikaner émerge ${ }^{23}$.

Ce nationalisme afrikaner se construit autour d'un certain nombre de représentations et notamment une vision différente de l'espace sud-africain. Celui-ci n'est pas, comme pour les anglophones, une nature sauvage à mettre en scène, mais une Terre (Land) qui leur est promise. Rurale, cette terre est mise en valeur et transformée par le travail d'un peuple paysan. Cette représentation alimentera une production littéraire et cinématographique qui s'apparente à celle des nationalistes allemands de l'Entre-deux guerres ${ }^{24}$.

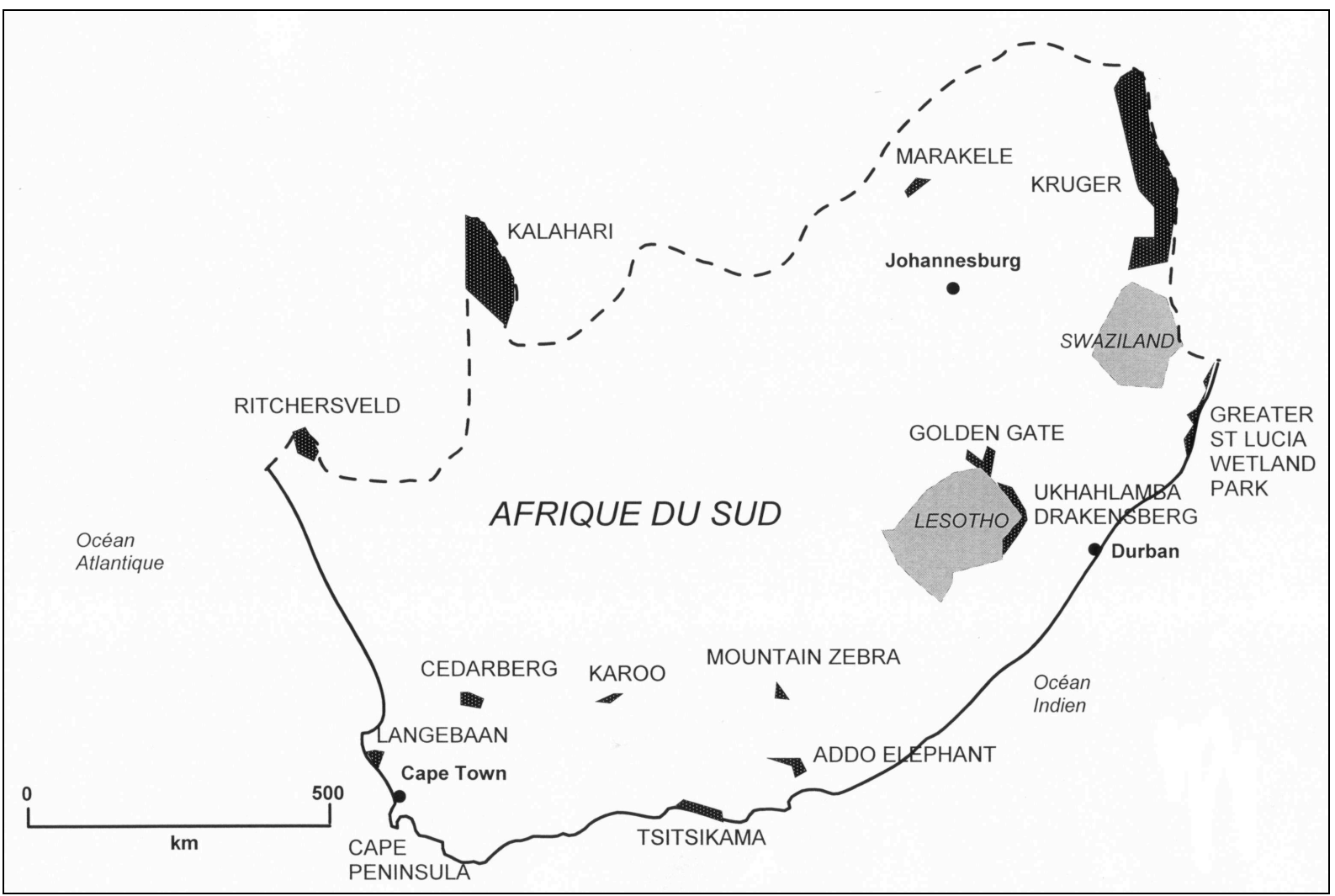

Figure 2 : Les parcs nationaux d'Afrique du Sud en 2005.

C'est d'ailleurs cette conception de l'Afrique du Sud qui permettra aux Afrikaners d'accéder au pouvoir en 1948 pour mettre en place leur programme d'apartheid. Une fois au pouvoir ils s'approprient symboliquement les parcs nationaux, attributs d'un État-Nation qu'ils dirigent désormais. Au cours des décennies suivantes, le conservationnisme va s'arrimer à un ensemble idéologique conservateur, comprenant pêle-mêle le nationalisme, la domination masculine, le sentiment de supériorité voire l'élitisme, la cause animale... En outre, cette approche s'appuie sur un malthusianisme inspiré du Club de Rome : la nature doit être protégée de la «prolifération » humaine - un raisonnement prenant en Afrique du Sud une résonance particulière dans la mesure où le péril démographique africain est l'un des fondements de la politique d'apartheid.

\footnotetext{
${ }^{22}$ Paul Kruger (1825-1904), président de la République du Transvaal pendant la guerre des Boers contre les Anglais, qui ne s'est jamais, dit on, intéressé à la faune sauvage autrement que sous la forme du biltong (viande séchée), proclama en 1898 une game reserve entre les rivières Sabie et Crocodile.

${ }^{23}$ J. Carruthers, « Nationhood and national parks... », art. cit.

${ }^{24}$ John M. Coetzee, White Writing: on the Culture of Letters in South Africa, New Haven, Yale University Press, 1988 ; A. Lester, From Colonization to Democracy. A new historical Geography of South Africa, London, I.B. Tauris Publishers, 1998.
} 
Cette conjonction politique entre conservationnisme et conservatisme explique que le champ politique de la nature ait été repris plus largement dans les décennies suivantes du régime d'apartheid. Ainsi, le nationaliste zoulou à la tête de l'Inkatha Freedom Party, Mangosuthu Buthelezi est très sensible à la question de la nature qui, pour lui, fait référence à une double expérience d'un passé glorieux : à la fois intime et élitiste (la réserve royale de faune) et collective (le territoire sauvage à protéger des envahisseurs).

"Both nationalist and environmentalist movements are 'interclassist', together they provide cosy refuges from the chilling winds of modernity that shatter people's identities. The link between conservation and ethnic nationalist notions of heritage is particularly strong in opposition: what is there to be proud of, if not the land and one's historical and cultural connection to it? Nationalism thrives on romanticism, not least romanticism about nature. ${ }^{.25}$

Par conséquent, il a toujours été très proche des grands acteurs de la conservation au KwaZulu-Natal, comme Nick Steele ${ }^{26}$, Ian Player $^{27}$ ou John Aspinall ${ }^{28}$, lors de la création dans le début des années 1980 du KwaZulu Bureau of Natural Resources.

Player's political experience in the local [conservationist] milieu took him in direction of Zulu ethnic nationalism. His 'Zululand Wilderness' is dedicated to Dr Mangosuthu Buthelezi and the late Nick Steele. Together they led the way to a new understanding of conservation in KwaZulu-Natal."29.

Cette coalition peut être à juste titre qualifiée de conservationniste et de très conservatrice. C'est à l'extrême droite de l'échiquier politique que se nouent ces collusions entre Zoulous et Anglophones. Le Zululand doit être réservé à une "ethnie zoulou", tout comme les Afrikaners ont le droit à leur Volkstaat et les droits sacrés et territoires des animaux doivent être respectés avant tout (mouvements de protection de la faune et contre la cruauté animale).

"The label of fascist has clung to John Aspinall ever since, notes his biographer admitting that Aspinall did little to disclaim it. (...)[In the beginning of the 1990's] he urged President de Klerk to 'abandon the election....and give the Afrikaners their Volkstaat and the Zulus and their English Speaking allies their own country in Natal...A greater Khosaland [sic] could include East London and no doubt Nelson Mandela will be its first president." ${ }^{30}$.

Certains acteurs environnementalistes au KwaZulu-Natal se réclament encore aujourd'hui de ce courant proche de celui des «écofascistes $»^{31}$.

\section{Des parcs et des réserves dans les constructions territoriales de l'apartheid}

Avec leur institution en cause internationale dans le monde anglo-saxon au début du siècle, les dispositifs d'aires protégés et notamment les parcs nationaux sont destinés à exister dans la durée comme sanctuaires naturels. Dès lors, ils vont disposer de régimes territoriaux spécifiques qui échappent aux pouvoirs locaux et vont être gérés par des organismes nationaux ou provinciaux qui disposent d'une autonomie, voire d'une quasi souveraineté sur

\footnotetext{
${ }^{25}$ M. Draper et G. Maré, “Going in: the Garden of England's Gaming Zookeeper and Zululand”, Journal of Southern African Studies, vol. 29, n², 2003, pp. 551-569.

${ }^{26}$ 1933-1997, directeur du KwaZulu Bureau of Natural Resources de 1983 à 1994.

${ }^{27}$ Né en 1927, ayant travaillé au Natal Parks Board, fondateur de la Wilderness Foundation. Voir M. Draper, "Zen and the Art of Garden Province Maintenance: the Soft Intimacy of Hard Men in the Wilderness of KwaZulu-Natal, South Africa, 1952-1997", Journal of Southern African Studies, vol. 24, n 4, 1998, pp. 801828 .

${ }^{28}$ Homme d'affaires britannique (1926-2000), aux conceptions eugénistes, propriétaire de zoo et de casinos, membre de l'IFP et ami de Margaret. Thatcher (ex premier ministre britannique). Voir M. Draper et G. Maré, "Going in: the Garden... », art. cit.

${ }^{29}$ M. Draper, "Zen and the Art of Garden Province Maintenance... », art. cit.

${ }^{30}$ M. Draper et G. Maré, "Going in: the Garden... », art. cit.

${ }^{31} \mathrm{Ph}$. Pelletier, L'imposture écologiste, op. cit. ; Sylvain Guyot, L'environnement contesté. La territorialisation des conflits environnementaux sur le littoral du KwaZulu-Natal (Afrique du Sud) : Kosi Bay, St Lucia, Richards Bay et Port Shepstone), Thèse de Doctorat, Université de Paris X/IRD, 2003.
} 
leur domaine. Dans le cas de l'Afrique du Sud, un National Parks Board est ainsi créé en $1926^{32}$.

Tout en relevant d'une dynamique internationale et d'une individualisation par rapport au système administratif, les aires protégées sud-africaines s'inscriront dans le dispositif toujours perfectionné de la ségrégation puis du « développement séparé » érigé en politique d'État à partir de la victoire du Parti National aux élections de 1948. Ainsi les gouvernements d'apartheid, à partir de cette date, ont adapté l'usage des parcs à leurs propres intérêts. La multiplication des campings et des autorisations de pêche a permis l'arrivée de nombreux touristes afrikaners, aux moyens souvent beaucoup plus modestes que ceux des anglophones.

Les parcs et réserves vont aussi apparaître comme un élément tardif de la fabrication des bantoustans dans le cadre de la politique du Grand apartheid. Conçus à partir de la fin des années 1950, les bantoustans doivent transformer le dispositif éclaté des réserves indigènes en différents cadres nationaux. Leur carte est élaborée progressivement tandis que des opérations de «consolidation» leur donne une configuration plus compacte que les lambeaux des réserves initiales, parallèlement les déplacements de population contribuent au peuplement dense de leurs marges. La marche vers une pseudo-indépendance s'effectue par une dotation progressive d'institutions autonomes (territorial authority, assemblée législative, selfgoverning body) puis souveraines («indépendance» accordée au Transkei, Ciskei, Bophuthatswana et Venda). Dans quelques cas, des «parcs nationaux » ou des réserves vont être créés comme attributs de la souveraineté nationale. Trois modalités peuvent être identifiées :

- Le legs initial d'une ressource, c'est le cas du Parc du Pilanesberg (actuel Marakele) créé pour doter le Bophuthatswana d'une ressource touristique périmétropolitaine que le futur complexe touristique de Sun City contribuera à valoriser ${ }^{33}$;

- La création de nouvelles aires protégées qui permettent d'une part d'étendre l'assise territoriale d'un bantoustan tout en introduisant une zone tampon à sa marge et d'autre part de contribuer à la mise place d'une politique de développement spécifique qui implique les populations rurales. C'est le cas du Parc National du QwaQwa ${ }^{34}$ créé en 1991 et issu d'une expropriation de fermiers blanc en bordure du Parc National du Golden Gate par les autorités sud-africaines dans les années 1980 ; c'est aussi le cas de la réserve de Manyeleti créé dans les années 1960 aux portes du Kruger sur des terres acquises par le South African Development Trust pour créer le Gazankulu et le doter d'une black game reserve ${ }^{35}$;

- La mise en place au KwaZulu d'un véritable complexe d'aires protégées (parcs d'Itala et de Tembe, réserve de Kosi Bay) doté d'une administration spécifique (le KwaZulu Board of Natural Ressources ou KBNR puis KwaZulu Directorate of Nature Conservation) à l'instar de celle du Natal avec laquelle les liens furent d'ailleurs étroits ${ }^{36}$.

\footnotetext{
${ }^{32}$ Il n'aura jamais autorité sur la Province du Natal qui cultive ici aussi son particularisme et conserve ses prérogatives d'abord au sein de l'administration provinciale puis avec le Natal Parks Game and Fish Preservation Board, organisme indépendant créé en 1947.

${ }^{33}$ J. Carruthers, « Nationhood and national parks... », art. cit.

${ }^{34}$ Il doit être rattaché en 2004 avec son Basotho Cultural Village au Golden Gate Highlands National Park et donc passer sous l'administration du South African National Parks.

${ }^{35} \mathrm{~K}$. Mahony et J. Van Zyl, "The impacts of tourism investment on rural communities: three case studies in South Africa”, Development Southern Africa, vol. 19, n¹, 2002, pp. 83-103.

${ }^{36}$ Benoît Rey-Soulingeas, La redéfinition des interfaces des parcs naturels au KwaZulu-Natal, Afrique du Sud, Mémoire de Maîtrise, Université de Grenoble I, 2001.
} 
Situés sur les marges frontalières (cf. figure 4), certains parcs sud-africains et namibiens vont occuper une fonction géopolitique de premier ordre, à une échelle cette fois internationale, dans les conflits qui embrasent l'Afrique australe à partir des années 1970, lorsque la guerre froide se projette dans la sous-région. Ils rejoignent une fonction classique des parcs nationaux frontaliers à la fois no man's land et terrains militaires qui permettent un contrôle étroit d'une ligne de front. Ainsi certaines parties du parc du Kruger ou encore la réserve de Ndumo ont été utilisées comme bases militaires d'entraînement et de soutien arrière à la RENAMO, guérilla mozambicaine s'opposant au FRELIMO, guérilla indépendantiste marxiste. La collusion entre la South African Defense Force et les agents du KBNR, entraînés comme force paramilitaire témoigne de cette tendance au sein même du bantoustan frontalier du KwaZulu ${ }^{37}$. Parallèlement, divers trafics vont se développer dans les parcs frontaliers, notamment le trafic d'ivoire qui enrichit alors les mouvements armés soutenus par le régime de Pretoria ainsi que des intérêts militaires sud-africains ${ }^{38}$. Ce dévoiement est facilité par le caractère traditionnellement paramilitaire de la protection de la nature en Afrique du Sud; il renforce en outre la perception répressive et violente que pouvaient en avoir les populations riveraines en butte aux rangers depuis la création de ces aires protégées.

Historiquement, les régimes en place entre 1890 et 1994 ont donc favorisé plusieurs modalités d'instrumentalisation politique de la nature. Outre des modalités politiciennes dans la constitution d'un bloc nationaliste et conservateur - et de représentations, les espaces naturels ont surtout servi d'outils pour une gestion territoriale de questions sociales. En d'autres termes, d'outils socio-territoriaux de la domination blanche.

\section{Le raccommodage sud-africain par la nature}

Depuis l'avènement de la démocratie en 1994, l'ingénierie territoriale de la nature en Afrique du Sud est encore un moyen privilégié du projet politique et social ${ }^{39}$. Ainsi, les objectifs gouvernementaux de réduction des inégalités s'appuient sur la promotion d'une gestion participative des ressources environnementales comme moyen privilégié de développement du monde rural. À l'échelle du sous-continent, l'ambition de dépasser les conflits récents et de constituer une entité régionale solidaire et prospère passe par la mise en place de parcs naturels transfrontaliers, les Transfrontier Peace Parks (TPP) ${ }^{40}$.

La révolution négociée sud-africaine ne va cependant pas traiter immédiatement de la question environnementale. D'une part, les enjeux sont évidemment plus vastes puisqu'il s'agit de mettre fin à la domination politique des Blancs sur le pays et aux inégalités qui en ont résulté. D'autre part, cela nous semble aussi marquer le succès de l'ingénierie territoriale de la nature pendant l'époque précédente : l'espace du naturel est, effectivement, sorti du

\footnotetext{
${ }^{37}$ Network of Independent Monitors, "Military Involvement in Conservation”, Crime and Conflict, n $10,1997$.

${ }^{38}$ S. Ellis, "Of elephants and men: politics and nature conservation in South Africa ", Journal of Southern African Studies, vol. 20, n¹, pp. 53-69, 1994.

39 Jean-Claude Fritz, «L'évolution des politiques de l'environnement en Afrique du Sud. Recomposition territoriale et protection de la nature », La nouvelle Afrique du Sud, Hérodote, n82-83, 1996, pp. 213-233 ; D.A. McDonald (dir.), Environmental Justice in South Africa, Cape Town, UCT Press, 2002.

${ }^{40}$ E. Koch, "Nature has the power to heal old wounds: War, peace \& changing patterns of Conservation in Southern Africa », in D. Simon (dir.), South Africa in Southern Africa, reconfiguring the region, David Philip, Cape Town, 1998, pp. 54-71 ; Maano Ramutsindela, «Glocalisation and Nature Conservation Strategies in 21st Century Southern Africa », Tijdschrift voor Economische en Sociale Geografie, vol. 95, n 1, 2004, pp. 61-72.
} 
territoire africain puisqu'il n'est pas un enjeu. La preuve en est que, dans le gouvernement d'unité nationale issu des premières élections démocratiques de 1994, le portefeuille de l'Environnement et du Tourisme est confié au Parti national. "Le ministère était dirigé par Dawie de Villiers, à la tête d'une vieille garde bureaucratique déjà en place ${ }^{41}$.

Pourtant, l'ANC (African National Congress) va instrumentaliser à son tour la nature sous la forme de l'enjeu environnemental. Plusieurs processus convergents vont le permettre :

- L'action de nombreuses ONG (Organisations Non Gouvernementales) ${ }^{42}$;

- L'action de Kader Asmal, ministre ANC des Eaux et Forêts, qui va faire de son ministère l'un des fers de lance de la reconstruction sud-africaine et souligner ainsi l'importance du levier environnemental pour la réduction des inégalités ;

- L'implication personnelle de Nelson Mandela dans des actions de protection de l'environnement, comme sa signature dès 1992 d'une pétition pour sauvegarder les dunes de St Lucia de l'extraction minière ${ }^{43}$;

- La multiplication des discours prônant une «justice environnementale» (environmental justice) et identifiant donc ce secteur comme devant faire partie d'une réflexion post-apartheid globale.

La fin du gouvernement d'unité nationale en 1996 avalisera ce changement idéologique puisque l'ANC prendra à sa charge le portefeuille de l'Environnement et du Tourisme ${ }^{44}$. Très vite, cette évolution dans le champ politique de l'environnement est intégrée dans le cadre d'une nouvelle politique économique globale et néo-libérale, GEAR (Growth, Employement And Redistribution): la nature est désormais un atout économique central pour le développement du pays, qui passe par le tourisme avec ses effets d'entraînement.

Par ailleurs, l'avènement de la démocratie a modifié radicalement les cadres de la pensée politique sud-africaine. La diversité de la société n'est plus niée, mais reconnue et promue : dans le Bill of Rights, dans l'officialisation du plurilinguisme (l'Afrique du Sud a onze langues officielles), dans le droit du travail avec une politique de discrimination positive, dans la volonté d'intégrer le droit coutumier et les "savoirs autochtones » à un état démocratique... Il s'agit donc d'une posture post-moderne explicite, voire revendiquée. Dans ce cadre, la nature est instrumentalisée dans une vision intégrant dialectiquement le global et le local. Cela se fait via la notion d'environnement, conçue à la fois comme :

- Une richesse à préserver, fond commun du discours écologisant international ;

- Un lieu de pratiques ancestrales respectueuses, que de nombreuses ONG développementalistes cherchent à reprendre dans un contexte de revalorisation des traditions africaines ;

- Une ressource à exploiter dans le cadre du développement durable - autre principe international très largement repris dans le contexte sud-africain.

Les différentes logiques historiques comme les multiples acceptions de la notion d'environnement vont se superposer et s'ajouter à un jeu entre les nombreux acteurs impliqués pour produire un enchevêtrement complexe. Nous les analyserons dans trois domaines qui constituent également trois échelles du « raccommodage » social et spatial entre les différentes communautés sud-africaines.

\footnotetext{
${ }^{41}$ D. Fig, "L'environnement en Afrique du Sud : l'Etat et la société civile face aux nouveaux enjeux", Revue Tiers Monde, 159 (XL), 1999, pp. 579-595.

${ }^{42}$ Anne Whyte, Building a new South Africa : environment, reconstruction and development, a report from the International Mission on environmental policy, Rapport technique, 1995.

${ }^{43} \mathrm{~S}$. Guyot, L'environnement contesté, op. cit.

${ }^{44}$ En 2004 pourtant, et en raison de considérations politiciennes, ce portefeuille revient à Marthinus Van Schalkwyk, chef du nouveau Parti national.
} 


\section{La participation ou le gouvernement local contourné}

Les limites intérieures créées par le green apartheid, la plupart du temps artificielles au regard des implantations historiques des populations, sont à l'origine d'un grand ressentiment chez les Noirs spoliés. Par conséquent, les revendications foncières - land claims - sont aujourd'hui très nombreuses et concernent notamment les parcs naturels ${ }^{45}$. Ce sont d'ailleurs celles sur les aires protégées qui connaissent des résolutions plus fortement médiatisées que celles portant sur les terres agricoles. Il y a là la possibilité de réparer symboliquement les injustices du passé dans la mesure où le gouvernement utilise parfois ces revendications foncières pour apparaître solidaire avec la majorité de la population tout en gardant un contrôle territorial fort sur les zones de nature. En effet, les rétrocessions partielles de terre et les compensations financières permettent souvent de calmer les exigences émanant des «communautés » et, ainsi, minimiser les conflits potentiels relatifs à une question foncière très sensible en Afrique australe.

Cette réparation symbolique s'opère a minima dans les changements de dénomination des aires protégées (Umkhahlamba rajouté à Drakensberg Park ; Malakele en lieu et place du Pilanesberg ou encore Kgalagadi Transfrontier Park plutôt que Kalahari) et des organismes gestionnaires (Natal Parks Board devient Ezemvelo KZN Wildlife). Plus profondément la question foncière peut faire l'objet de conciliations sur des terrains du domaine public (étatiques ou provinciaux), sans en changer réellement l'usage. En effet, dans la plupart des cas, il s'agit d'offrir des compensations financières en associant les communautés spoliées (par la chefferie ou individuellement) aux bénéfices tirés de l'exploitation touristique. Sur un plan foncier, le transfert aux communautés riveraines des droits sur tout ou partie de l'aire protégée reste associé à une contrainte d'usage : elle doit rester un parc ou une réserve, avec obligation de protection selon les normes internationales et ouverture aux touristes...

De plus, la répartition des aires protégées dans l'ensemble du pays permet d'opérer cette redistribution symbolique dans toutes les provinces. Cela au profit de populations qui incarnent la diversité et l'historicité de la nation «arc-en ciel». La satisfaction des revendications de la communauté Venda de Makuleke sur une aire du complexe protégé du Kruger $^{46}$ et de la communauté gKhomani San aux abords du Kalahari Gemsbok National Park ont été les plus médiatisées. Dans ce dernier cas, l'influence du statut nouveau des «peuples premiers » sur le modèle australien est visible. Il s'agit ici d'une communauté de quelques centaines de membres, comprenant les derniers locuteurs de la langue $\mathrm{N} / \mathrm{u}$. Cette communauté, initialement incluse dans le parc en 1931, puis considérée comme coloured sous l'apartheid, avait été évincée de l'aire protégée en direction de la location voisine dans les années $1970^{47}$. La communauté zouloue de Bhangazi au nord de St Lucia dans la province du KwaZulu-Natal ${ }^{48}$ a lancé une procédure de land claim résolue en 2000, par une commission

\footnotetext{
${ }^{45}$ Greater St Lucia, Wetland Park, Ndumo Game Reserve, Tembe Elephant Park, Kruger National Park, Richtersveld...

${ }^{46}$ Maano Ramuntsindela, Unfrozen Ground: South Africa's Contested Spaces, Aldershot, Ashgate, 2001 ; Maano Ramuntsindela, "Down the post-colonial road: reconstructing the post-apartheid state in South Africa", Political Geography, 20, 2001, pp. 57-84; B. N. Tapela, P.H. Omara-Ojungu, "Towards bridging the gap between wildlife conservation and rural development in post-apartheid South Africa: the case of Makuleke community and the Kruger national Park”, South African Geographical Journal, vol. 81, n³, 1999, pp. 148-155.

${ }^{47}$ J. Cock, D. Fig, "From Colonial to Community-Based Conservation: Environmental Justice Discourse" in D.A. McDonald (dir.), Environmental Justice in South Africa, Cape Town, UCT Press, 2002, pp. 131-155.

${ }^{48}$ La plupart de ces espaces, composés de prairies ou de forêts, sont devenus entre 1956 et 1964 des State Forest (Cape Vidal State Forest, Eastern Shores State Forest) dédiés à l'exploitation forestière et non pas à la
} 
d'arbitrage moyennant une compensation financière de la part du Department of Water Affairs and Forestry ainsi que le legs de 4 ha du World Heritage Site du Greater St Lucia Wetland Park, près du Lake Bhangazi, pour y développer un lodge. Une cérémonie de commémoration à la gloire des ancêtres pourra ainsi avoir lieu chaque année, à la fois attraction touristique et symbole d'une réappropriation patrimoniale ${ }^{49}$.

Ces restitutions sont à interpréter selon deux axes complémentaires. Le premier intègre l'Afrique du Sud post-apartheid dans des dynamiques internationales, consistant à appliquer de nouveaux principes de gestion des aires protégées tournés vers la participation des peuples « indigènes et traditionnels ». C'est la priorité de l'Union Internationale de la Conservation de la Nature ${ }^{50}$.

Un second axe d'interprétation est plus spécifique au contexte sud-africain et particulièrement à celui du KwaZulu-Natal. Il s'agit d'utiliser ces directives internationales pour repenser la structure du gouvernement local ${ }^{51}$, pour y faire participer des communautés africaines longtemps exclues de la vie politique et les faire bénéficier des revenus générés par les aires protégées se trouvant sur leur territoire. Des institutions établies par l'administration provinciale des Parcs, les Local Boards, vont dans ce sens. Ils instaurent une scène d'échange et de négociation entre chaque parc et les communautés environnantes. Ces conseils sont constitués d'un côté de représentants du Ezemvelo KZN Wildlife et de l'autre de membres nommés par la Province et censés représenter : les autorités traditionnelles; les secteurs de l'agriculture, du tourisme et du commerce ; les associations de défense de l'environnement ; le gouvernement local et tout "groupe représentant des intérêts particuliers". Après quelques années de fonctionnement, le bilan est pour l'instant contrasté. Si une dynamique positive semble s'instaurer autour du parc de Hluhluwe via un collectif d'autorité tribale qui gère des revenus en augmentation pour promouvoir des infrastructures touristiques en cogestion, en revanche la situation dans les confins septentrionaux de la province (Kosi Bay ou Tembe) est nettement plus conflictuelle. On y note d'une part l'attitude rentière de certains chefs qui sont rétifs à un changement de règle pour un usage plus collectif des fonds ${ }^{52}$ issus des parcs et réserves, et d'autre part un manque évident de coordination avec les nouvelles autorités municipales et un vaste projet d'aménagement relevant de la procédure nationale de Spatial Development Initiative ${ }^{53}$. On touche là une des contradictions majeures de l'instauration de micro-structures participatives sur le pourtour des parcs dans un contexte de promotion du gouvernement local qui s'appuie sur des légitimités politiques et spatiales liées à la

conservation de la nature. 5000 personnes vivant dans les Eastern Shores furent alors expulsées et une partie d'entre elles relogée à Mbazwana dans le futur bantoustan. Les membres de la communauté des Eastern Shores est maintenant très dispersée et l'argent du dédommagement va profiter à des familles habitant à Durban ou à Johannesburg.

${ }^{49} \mathrm{~S}$. Guyot, L'environnement contesté, op. cit.

${ }^{50}$ Lors du IVe Congrès mondial sur les parcs nationaux et les aires protégées (Caracas, Venezuela, 1992) il fut ainsi recommandé l'élaboration de politiques sur les aires protégées qui tiennent compte des intérêts des peuples autochtones, des pratiques coutumières liées aux ressources et des systèmes traditionnels de régime foncier. En octobre 1996, c'est le Congrès mondial de la nature réuni à Montréal qui adopte une résolution sur les «Principes et lignes directrices sur les peuples autochtones et traditionnels et les aires protégées », enfin le thème/slogan du récent congrès mondial des parcs de Durban en 2003 s'intitulait «Benefits beyond boundaries ».

${ }^{51}$ S. Parnell, E. Pieterse, M. Swilling, D. Wooldridge (dir.), Democratising Local Government: the South African Experiment, Cape Town, UCT Press, 2002.

${ }^{52}$ Community levies dont une part était versée directement et sans contrôle aux chefs des autorités tribales riveraines.

${ }^{53}$ S. Luckett, K. Mkhize, D. Potter, “The experience of Local Boards in KwaZulu-Natal, South Africa”, Parks, vol. $13, \mathrm{n}^{\circ} 1,2003$, pp. 6-15. 
démocratie représentative ${ }^{54}$ mais doit tenir compte des héritages passés encore solidement ancrés dans l'espace et les mentalités.

\section{L'unité nationale contre les Alien Plants}

Malgré ces nouveaux modes de gouvernance environnementale, peu médiatisés en dehors des espaces de parcs et des réserves, on constate que la nature est souvent absente des discours officiels sud-africains. Cependant, un thème bien particulier semble faire exception à cette règle: la question des alien plants ou plantes allochtones, nouvel exemple d'instrumentalisation de la nature à des fins politico-idéologiques.

Il s'agit de plantes qui ne sont pas autochtones et qui ont colonisé le paysage végétal au gré des migrations et colonisations successives. De nombreuses campagnes d'information et une grande mobilisation de moyens financiers publics et privés, ainsi que des politiques publiques, ont visé ces plantes allochtones. Les premières actions contre les alien plants ont d'abord été menées par les autorités de conservation nationale et provinciales dans les parcs et réserves du pays et par des associations écologistes conservatrices comme WESSA (Wildlife and Environment Society of South Africa). Un grand programme national, Working for Water, initié en 1995 par le Ministère des Eaux et Forêts de Kader Asmal (cf. supra), est motivé par un triple constat :

- «Les plantes allochtones touchent plus de $8 \%$ de l'étendue du pays. Elles se répandent à un rythme alarmant, nuisent à la croissance de la végétation naturelle et mettent en péril l'équilibre écologique en augmentant les incendies et en favorisant l'érosion.

- Ces plantes ont toujours soif. Elles réduisent donc grandement les nappes d'eau souterraines et assèchent les cours d'eau. C'est un problème dans un pays périodiquement menacé de sécheresse où 12 millions de Sud-africains n'ont pas accès à l'eau potable.

- Ce programme cherche à créer des emplois (initiative sociale) dans un pays où la pauvreté, le chômage et l'analphabétisme sont généralisés.» ${ }^{55}$

On le voit, le discours politique est extrêmement alarmiste et instaure un lien puissant entre nature (espèces végétales ici) et développement. L'ambiguïté de la perception de la Nature sud-africaine - même si le terme de nature est un peu exagéré ici - est toujours présente : ce n'est plus l'ancienne représentation oscillant entre Eden à préserver et jungle à domestiquer, mais bien une vision post-moderne. La Nature est désormais comprise, dans les discours politiques sud-africains, comme un élément toujours à la fois fragile et dangereux, mais vis-à-vis duquel l'homme a une double responsabilité : passée (l'introduction des plantes allochtones) et future (protéger une ressource en eau menacée). Cette responsabilité est un appel général à l'action puisque Working for Water cherche à fédérer les «énergies destructrices » de plantes allochtones dans tout le pays.

Ces messages politiques ont donc véhiculé un discours important : les alien plants sont indésirables et même dangereuses pour l'Afrique du Sud. Il convient donc de les éradiquer

\footnotetext{
${ }^{54}$ Benoît Antheaume, Frédéric Giraut, « Les marges au cœur de l'innovation territoriale ? Regards croisés sur les confins administratifs (Afrique du Sud, France, Maroc, Niger, Togo...) », in F. Bart, J. Bonvallot, R. Pourtier (dir.), Historiens et géographes, $\mathrm{n}^{\circ}$ spécial : Regards sur l'Afrique, 2002, pp. 39-58 ; R.P. Neumann, "Primitive Ideas: Protected area buffer zones and the politics of land in Africa", Development and Change, 28, 1997, pp. 559-582.

${ }^{55}$ WFW, 2003, Working for Water : l'éradication des plantes non indigènes en Afrique du Sud. www.idrc.ca
} 
pour empêcher leur pouvoir de nuisance. Toute la population (tous groupes confondus) est invitée à y participer (cf. figure 3).

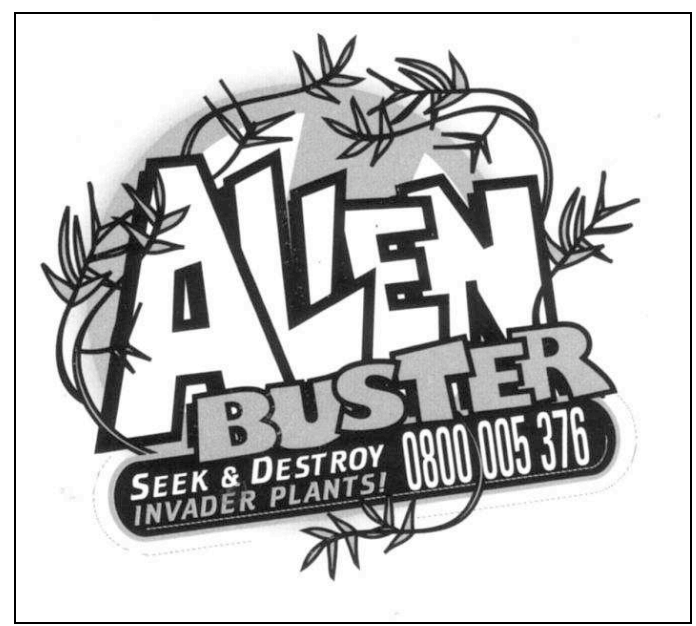

Figure $3:$ : Be an Alien Buster \& Win »

Logo et slogan d'une campagne nationale sud-africaine en 2001

Ce logo de la campagne apparaissait sur des affiches, des autocollants et des guides pédagogiques largement distribués dans la population en 2001 par le DWAF ${ }^{56}$ via les écoles. Il est révélateur d'un discours, de ses connotations voire de ses dérives. C'est le terme d'Alien qui apparaît au premier regard. Il convient de noter que ce terme sert aussi à désigner toute personne immigrée récemment en Afrique du $\mathrm{Sud}^{57}$. Dans un pays xénophobe, combattre les étrangers - même si l'on se rend compte ensuite qu'il s'agit de végétaux - est une injonction qui peut sembler ambiguë. Cela est amplifié par l'usage d'un vocabulaire militaire spécifique, appartenant aux opérations commandos. Enfin, le graphisme menaçant des plantes renforce l'effet recherché.

L'importance donnée en Afrique du Sud à cette question est très révélatrice de la collusion entre un mode purificatoire du traitement de la nature et des idéologies politiques extrémistes que l'on retrouve dans d'autres «pays neufs» comme les États-Unis ou l'Australie. Les campagnes contemporaines contre les alien plants puisent en fait leurs origines idéologiques dans l'eugénisme et le désir de puretée ${ }^{58}$. En Afrique du Sud, ce processus exemplaire « recycle » donc l'idéologie raciste de l'apartheid dans un combat écologiste ouvert à tous. De plus elle s'inscrit bien dans les politiques post-1994 de réconciliation, car les nouveaux coupables de la dégradation socio-économique ne sont plus ni les Blancs ni les Noirs mais des cortèges de plantes allochtones. Cette action permet donc surtout de rallier les différents groupes de population, "races, sexes et âges confondus ${ }^{59}$ constitutif d'un «nous », collectif national rassemblé face à la menace végétale. Là encore, la nature est politiquement instrumentalisé pour servir un double but politique, celui de la construction nationale et du développement.

\footnotetext{
${ }^{56}$ Department of Water Affairs and Forestry.

57 J. Comaroff, J.L. Comaroff, "Naturing the Nation: Aliens, Apocalypse and the Postcolonial State", Social Identities, vol. 7, $\mathrm{n}^{\circ} 2,2001$, pp. 233-265.

${ }^{58} \mathrm{Ph}$. Pelletier, L'imposture écologiste, op. cit.

${ }^{59}$ WFW, 2003, Working for Water : l'éradication des plantes non indigènes en Afrique du Sud. www.idrc.ca

${ }^{59}$ www.dwaf.org.za, 2003
} 


\section{Des peace parks pour construire un continent}

Les Transfrontier Conservation Areas de la Peace Parks Fundation offrent une troisième échelle, transnationale, de l'utilisation de la nature pour répondre aux défis postapartheid. Là encore, les ambiguïtés de la démarche montrent que les discours sur la nature restent complexes et contradictoires.

Plus de 50 ans après une première tentative avorté ${ }^{60}$, les espaces naturels protégés sont utilisés pour promouvoir l'intégration régionale en Afrique australe, multiplier les coopérations entre pays par une politique commune de conservation et de développement via le tourisme. Le moyen de cette intégration est la mise en place de « super parcs » naturels, réunissant les parcs préexistants de chaque côté des frontières nationales. Il s'agit donc de construire des objets internationaux en lieu et place d'anciens hauts-lieux des divisions régionales entre le régime de l'apartheid et ceux des États de la Ligne de front. Un tel procédé peut concerner plusieurs pays : ainsi, le Great Limpopo Transfrontier Park réunit le parc Kruger sud-africain, le parc de Gaza au Mozambique et Gonarezhou au Zimbabwe. De nombreux acteurs sont impliqués dans de telles constructions :

- Des acteurs supranationaux institutionnels : l'UICN (Union Internationale pour la Conservation de la Nature) a recommandé la mise en place de telles structures et identifié les zones potentiellement concernées en 1988 ;

- Des acteurs supranationaux non gouvernementaux, comme le World Wildlife Fund (WWF), à partir de 1990, par la voix du président de WWF-Afrique du Sud, Anton Rupert, qui cherche à restaurer le fonctionnement « originel »- non gêné par l'activité humaine - de l'écosystème, et notamment des migrations des grands mammifères ;

- Les États de la région.

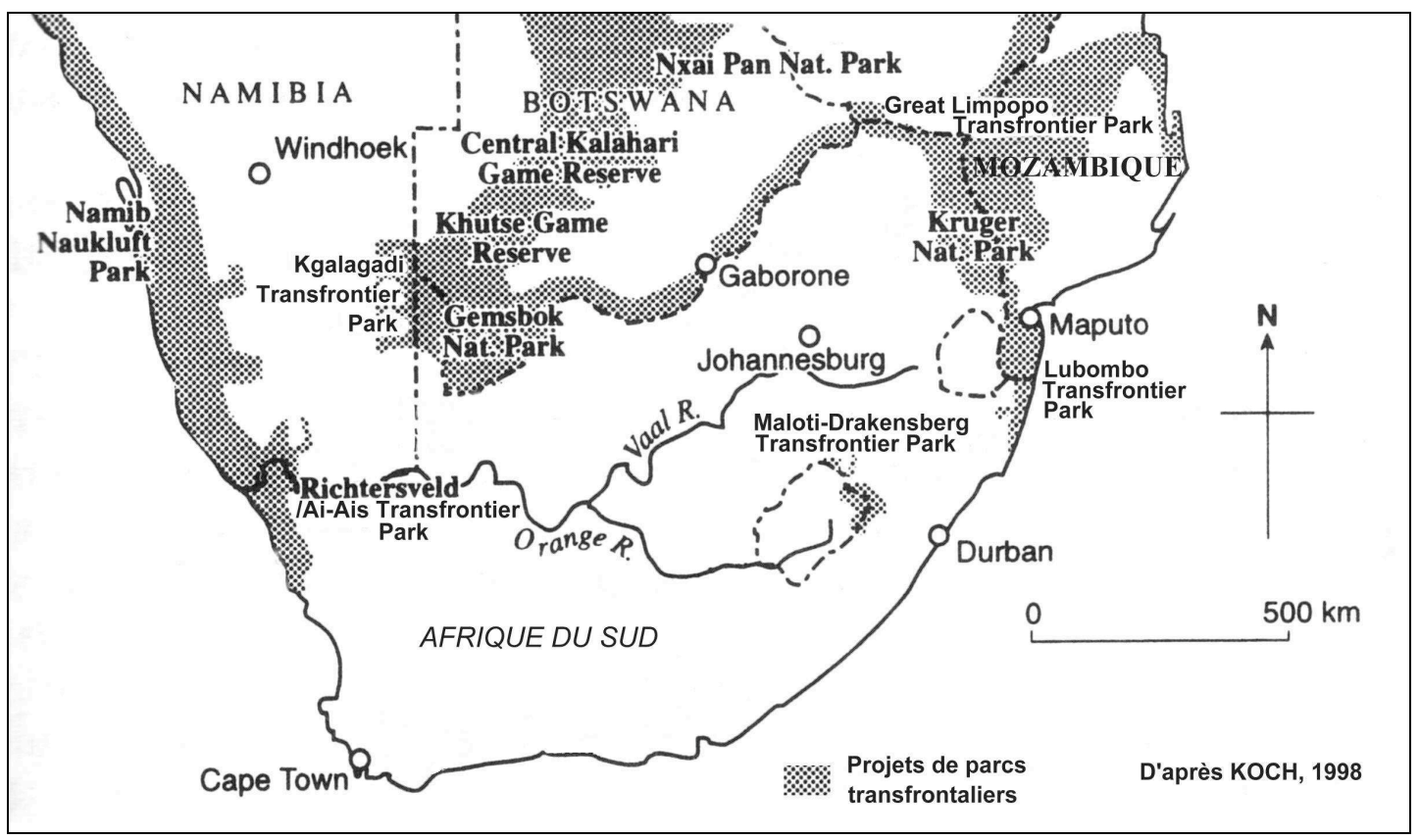

\footnotetext{
${ }^{60}$ Jane Carruthers (1997) rappelle l'épisode de l'éphémère Parc de Dongola. Premier parc international et premier parc non exclusivement animalier créé en 1947 sur la frontière septentrionale avec le Bechuanaland et la Rhodésie, il fut déclassé après l'avènement du Parti National en 1948. Ses opposants contestaient le gel d'intérêt miniers et fonciers mais aussi le caractère international du projet.
} 
Figure 4 : Le projet de dispositif de parcs transfrontaliers en Afrique australe ${ }^{61}$

Les Transfrontier Peace Parks (TPP) ancrent donc l'image de l'Afrique australe comme zone majeure et innovante de la conservation de la nature dans le monde. Cela est en accord avec les discours tenus localement et internationalement sur le développement durable. De plus, les aspects sociaux, fondateurs de la politique post-apartheid, sont également présents : on met, bien sûr, l'accent sur le développement local engendré par le tourisme à venir et la gestion participative, garante de bonne gouvernance... Tous les mots-clefs des discours politiquement corrects susceptibles de motiver des bailleurs de fonds internationaux sont donc présents. La Banque mondiale, sollicitée par le Mozambique pour financer les TPP, a effectivement apporté son soutien.

Les réalisations concrètes sont encore faibles. Le TPP du Kalahari (figure 4) rebaptisé Kgalagadi lors de son inauguration en 1999, est le premier à être mis en place. Il est donc trop tôt pour juger de leur efficacité réelle. Nous pouvons cependant souligner d'emblée que cette initiative apparemment nouvelle recycle des discours et des acteurs de la période précédente. Par exemple, Anton Rupert, qui apparaît ici comme le représentant bienveillant du WWF, est l'ancien PDG de la compagnie de tabac Rembrandt puis de Rothmans International. À ce titre, il a longtemps été le symbole de l'homme d'affaires afrikaner, richissime, extrêmement influent dans les cercles du pouvoir. Il a été membre du Broederbond, société secrète afrikaner dont la mission était de coordonner les activités de l'élite politique et économique afrikaner en vue de maintenir leur domination sur le pays. De plus, les discours justifiant les TPP se fondent sur une vision néo-coloniale du continent, comme « the greatest animal kingdom $»^{62}$, terre de nature où les animaux vagabondent librement. Les hommes n'y sont présents que marginalement, sous trois figures principales : celle, globale, du touriste ; celle, folklorisé, de l'Africain présenté dans des «cultural villages » ${ }^{63}$; celle, médiatrice et experte, du ranger.

De plus, les TPP participent d'un contexte géopolitique particulier. Ils ont été rendus possibles par la fin de la guerre au Mozambique et la fin de l'apartheid : les frontières se sont alors ouvertes ; la coopération entre les différents gouvernements, et entre les gouvernements et les instances internationales est devenue possible. En même temps, ce contexte géopolitique a aussi rendu possible le développement d'une hégémonie sud-africaine à l'échelle de l'Afrique australe - voire de l'Afrique subsaharienne. Cette domination est politique, économique, mais aussi touristique et «environnementale » : les TPP sont aussi un moyen de cette politique de puissance dans la mesure où ils renforcent le rôle central de l'Afrique du Sud. Les touristes internationaux, arrivant à Johannesburg, pourront visiter les principaux parcs naturels des pays voisins tout en restant basés en Afrique du Sud. L'arrièrepays touristique a simplement été étendu à une partie des pays voisins.

\section{Conclusion}

\footnotetext{
${ }^{61}$ E. Koch, « Nature has the power... », art. cit.

${ }^{62}$ Ironiquement, l'expression est de Valli Moosa, ministre ANC de l'Environnement sous la première présidence de Thabo Mbeki.

${ }^{63}$ M. Draper, H. Wels, « Super African Dreams: The Mythology of Community Development in Transfrontier Conservation Areas in Southern Africa », Séminaire Ecotourism and Nature Parks in East and Southern Africa, African Studies Center, Leiden, 2002.
} 
La nature sud-africaine est donc depuis longtemps instrumentalisée pour appuyer les différents projets politico-territoriaux des pouvoirs en place. Si la mise en place de réserves naturelles et de parcs a servi à confirmer la domination blanche, les usages actuels replacent ces espaces dans des logiques post-apartheid, aux discours généreux et aux pratiques ambiguës. La nature protégée se caractérise aujourd'hui par des paradoxes. D'un côté, c'est un outil symbolique de réparation à trois échelles : régionale avec des land claims réussies mais virtuelles ; nationale avec la construction d'une unité face à la «menace » des alien plants; et internationale avec la valorisation d'une ressource partagée en lieu et place des lignes de front. De l'autre, la conservation de la nature reste un domaine sectoriel réservé, comme en témoignent la nomination du dirigeant du New National Party (héritier direct du Parti national, détenteur du pouvoir sur toute la période d'apartheid) comme nouveau ministre de l'environnement et le recyclage d'un discours conservationniste intégriste en conjonction avec des thèmes comme le néo-libéralisme, la bonne gouvernance et le développement local et durable.

En fait, se trouve ici exacerbée une problématique continentale, voire universelle : les aires protégées deviennent un champ privilégié du développement communautaire participatif qui s'appuie sur les pouvoirs locaux dits « traditionnels » ou « coutumiers » détenteurs d'une approche collective de la propriété et des usages ${ }^{64}$. Ce choix s'oppose ainsi souvent à la légitimité démocratique des collectivités locales, porteuse d'une autre vision de la citoyenneté et du projet politique. Il est là question d'échelle dans le partenariat : la communauté locale, souvent villageoise, est privilégiée au détriment du gouvernement local assis sur une circonscription plus vaste ; mais il est aussi question de configuration socio-spatiale : la ou les communautés périphériques dépendantes d'un milieu plutôt que la société locale inscrite dans un bassin fonctionnel polarisé sur une ville ou un bourg. On peut bien sur se demander dans le cas de l'Afrique du Sud si ces communautés rurales ciblées et leurs représentants « traditionnels » sont bien représentatifs des noirs marginalisés qu'ils sont parfois censés incarner dans les discours. Si l'on reprend la thèse de Neumann (1997) sur l'extension des parcs nationaux africains via leurs zones périphériques comme moyen pour les Etats d'accroître leur domaine de contrôle territorial exclusif, on peut qualifier les nouveaux partenariats à la périphérie des parcs et réserves de moyen déguisé pour les Etats de contourner la décentralisation par la remise en selle d'autorités « coutumières ». Nouvelle version localisée du célèbre "divide and rule", cette vieille technique coloniale se déploie cette fois au nom des savoirs traditionnels, de l'autochtonie et du développement durable.

Loin d'être seulement un instrument idéal d'unification sociale et spatiale, la nature et ses usages contemporains peuvent aussi continuer à diviser, exclure et produire une géographie conflictuelle. En Afrique du Sud plus qu'ailleurs, cette contradiction doit être relevée. N'y voit-on pas finalement le temps du raccommodage à la marge, troisième acte d'une longue histoire chaotique de l'instrumentalisation territoriale de la nature après « le temps colonial des réserves » puis celui du « Green apartheid »?

\footnotetext{
${ }^{64}$ D. Compagnon, F. Constantin (dir.), Administrer l'environnement en Afrique, Paris/Nairobi, Karthala/IFRA, 2000 ; Est. Rodary, Les espaces naturels..., op. cit.
} 